\begin{tabular}{|l|l|l|}
\hline \multicolumn{2}{|c|}{ PublisherInfo } \\
\hline \hline PublisherName & $:$ & BioMed Central \\
\hline \hline PublisherLocation & $:$ & London \\
\hline \hline PublisherImprintName & $:$ & BioMed Central \\
\hline \hline
\end{tabular}

\title{
Cathepsin S null mice exhibit relative resistance to collagen- induced arthritis
}

\begin{tabular}{||l|l|l||}
\hline \multicolumn{2}{|c||}{ ArticleInfo } \\
\hline \hline ArticleID & $:$ & 243 \\
\hline \hline ArticleDOI & $:$ & $10.1186 /$ ar-1999-66728 \\
\hline \hline ArticleCitationID & $:$ & 66728 \\
\hline \hline ArticleSequenceNumber & $:$ & 200 \\
\hline \hline ArticleCategory & $:$ & Paper Report \\
\hline \hline ArticleFirstPage & $:$ & 1 \\
\hline \hline ArticleLastPage & $:$ & 4 \\
\hline \hline & & RegistrationDate : 1999-9-3 \\
ArticleHistory & $:$ & OnlineDate \\
\hline \hline ArticleCopyright & $:$ & Current Science Ltd1999-9-3 \\
\hline \hline ArticleGrants & $:$ & \\
\hline \hline ArticleContext & $:$ & 130753311 \\
\hline \hline
\end{tabular}




\section{Keywords}

cathepsin S, collagen-induced arthritis, CIA

\section{Context}

MHC class II molecules present T cell epitopes from exogenous (foreign) proteins as well as from endogenous (self) proteins destined for expression on the cell surface or for secretion. Class II molecules are protected from inappropriate peptide loading during their maturation by association with the invariant chain (Ii). The invariant chain is progressively degraded by a series of proteases until only fragments around residues 81-104, termed CLIP, remain to block the peptide-binding groove of MHC class II molecule. CLIP is later replaced by a diverse array of peptides derived from exogenous and endogenous proteins; this process is catalysed by HLA-DM. Lysosomal proteases, known as cathepsins, are involved in the degradation of both Ii and protein antigens. Recently, cathepsin L has been demonstrated to play an important role in processing Ii in cortical thymic epithelial cells but not in peripheral antigen-presenting cells. Studies with recombinant cathepsin S (CS) and with specific inhibitor of CS suggested that this protease may be necessary for Ii degradation in B cells, dendritic cells (DC) and macrophages (Mph). The absence, or variation in function, of a lysosomal protease may alter the repertoire of T cell epitopes presented by a class II MHC molecule and may influence the immune response to both self or foreign antigens. To investigate the effect of isolated CS deficiency on antigen processing and presentation in the context of MHC class II molecules.

To compare the severity of collagen-induced arthritis (CIA) in wild-type and CS-deficient mice.

\section{Significant findings}

The $\mathrm{T}$ and $\mathrm{B}$ cell compartments of CS-/- mice were grossly normal and the expression of MHC class II (either I-Ab or I-Aq) was not significantly decreased. In both splenic B cells and DCs from CS-/- I-Ab mice, there was a marked reduction of SDS-stable I-A dimers, compared with CS $+/+$ mice. Pulse-chase experiments revealed an accumulation of I-Ab bound to Ii degradation intermediates at both $3 \mathrm{~h}$ and $6 \mathrm{~h}$ after pulsing with radiolabelled methionine and cysteine residues. Unlike B cells and DCs, peritoneal Mph express cathepsin L as well as CS and, in CS-/- mice, the processing of Ii appeared relatively 
normal. In contrast with I-Ab CS-/- mice, B cells and DC from I-Aq CS-/- mice generated SDS-stable IA complexes normally and there was no accumulation of Ii degradation intermediates.

Assessment of the presentation of peptides from endogenous and exogenous antigens to $\mathrm{T}$ cell hybridomas revealed subtle, antigen- and antigen presenting cell (APC)- specific defects in T cell responsiveness. Surprisingly, the I-Aq CS-/- mice exhibited a selective inability to present collagen type II epitope (residues 260-270) to a specific T cell hybridoma. Attempts to induce CIA in CS-/- I-Aq mice revealed a reduced incidence and severity of disease, compared to I-Aq wild-type mice. This was paralleled by decreased production of anti-collagen type II antibodies.

\section{Comments}

In autoimmune or infectious/autoimmune diseases, such as RA or Lyme disease, the goal of immunotherapeutics is to selectively inhibit pathologenic immune responses, while leaving the patient otherwise immunocompetent. Although the CS-/- murine model is encouraging, the therapeutic application of cathepsin blockade presents several problems. The influence of blockade on presentation of a given antigen is, at present, unpredictable and, in certain cases, $\mathrm{T}$ cell responses were enhanced rather than diminished. In addition, the effect of CS deficiency was MHC class II allele-specific and it will be necessary to study the effect of CS blockade on a range of human T cell epitopes and class II molecules, in order to predict the therapeutic efficacy. Nevertheless, the prospect of cathepsin blockade is attractive, and this strategy could rationally be applied in diseases where particular target epitopes or MHC class II molecules are implicated in pathogenesis.

\section{Methods}

CS-deficient mice were generated by gene targeting and embryonic stem cell technology. The CS-/phenotype was bred with DBA/1 J (I-Aq) and C57Bl/6 (I-Ab) mice and antigen processing and presentation by these I-A molecules were characterised. The presence of grossly normal $\mathrm{T}$ and $\mathrm{B}$ cell compartments was verified by FACS (fluorescence-activated cell sorter) analysis and the kinetics of Ii processing was investigated in pulse-chase experiments using the following antibodies: In-1 (specific for the N-terminal region of murine Ii), AF6 (anti-I-Ab), KH116 (anti-I-Aq), 15G4 (specific for small Ii fragments: CLIP or short leupeptin protein). The presence of SDS-stable I-A alpha/beta dimers (indicative of mature, peptide-loaded class II molecules) was assessed by SDS-PAGE.The presentation of peptides from both endogenous and exogenous proteins was assessed using a panel of $\mathrm{T}$ cell hybridomas restricted to either I-Ab or I-Aq; stimulation of the hybridomas was assessed by measuring their secretion of interleukin (IL)-2.The influence of CS deficiency on the induction and severity of CIA was assessed by immunizing groups of male wild-type and CS-/- mice with chick collagen type II in complete Freund 


\section{Additional information}

In the same issue of Immunity, an independent group published their results from studies of I-Ab CS deficient mice (Shi et al 1999, abstract, 10:197-206). This group did not study multiple class II alleles or a disease model. However, they report similar impairment of Ii degradation in CS-/- mice and variable diminution or enhancement of presentation of $\mathrm{T}$ cell epitopes from exogenous proteins.

\section{References}

1. Nakagawa TY, Brissette WH, Lira PD, Griffiths RJ, Petrushova N, Stock J, McNeish JD, Eastman SE, Howard ED, Clarke SR, Rosloniec EF, Elliott EA, Rudensky AY: Impaired invariant chain degradation and antigen presentation and diminished collagen-induced arthritis in cathepsin S null mice. Immunity. 1999, 10: 207-217.

This PDF file was created after publication. 